\title{
Impact of size mismatch induced quenched disorder on phase fluctuation and low field magnetotransport in polycrystalline $\mathrm{Nd}_{0.58-x} \mathrm{Gd}_{x} \mathrm{Sr}_{0.42} \mathrm{MnO}_{3}$
}

\author{
Manoj K. Srivastava, ${ }^{1}$ Ravikant Prasad, ${ }^{1}$ P. K. Siwach, ${ }^{1}$ M. P. Singh, ${ }^{2}$ and H. K. Singh ${ }^{1, a)}$ \\ ${ }^{1}$ National Physical Laboratory (CSIR), Dr. K. S. Krishnan Marg, New Delhi-110012, India \\ ${ }^{2}$ Department of Materials Science and Engineering, University of Washington, Seattle, Washington \\ 98195-2120, USA
}

(Presented 19 January 2010; received 31 October 2009; accepted 2 December 2009; published online 12 May 2010)

\begin{abstract}
We report the magnetic and transport properties of polycrystalline $\mathrm{Nd}_{0.58-x} \mathrm{Gd}_{x} \mathrm{Sr}_{0.42} \mathrm{MnO}_{3}(x \sim 0.0$, $0.04,0.08,0.12,0.16,0.20,0.25,0.30,0.35$, and 0.42). All the samples are single phase and have grain size $\sim 1-2 \mu \mathrm{m}$. As the variance $\sigma^{2}$ increases, the paramagnetic-ferromagnetic transition shows a gradual decrease and broadening, while the decrease in insulator metal transition is sharpened. However, near equality of $T_{C}$ and $T_{\mathrm{IM}}$ at intermediate values of $\sigma^{2}$ could be understood in terms of the competing quenched and the grain boundary disorder. The variation in peak magnetoresistance (MR) (maximum MR around $T_{C} / T_{\mathrm{IM}}$ ) with $\sigma^{2}$ shows that maximum low field $\mathrm{MR} \approx 35 \%$ at $H=3 \mathrm{kOe}(68 \%$ at $H=10 \mathrm{kOe})$ is centered around $\sigma^{2}=0.009857 \AA^{2}(x=0.25)$. This shows that huge intrinsic MR can be obtained at relatively higher temperatures and lower magnetic fields in the region of enhanced phase fluctuations. (c) 2010 American Institute of Physics. [doi:10.1063/1.3358618]
\end{abstract}

\section{INTRODUCTION}

Phase coexistence due the occurrence of several magnetoelectronic phases, such as paramagnetic insulator, ferromagnetic metal (FMM), ferromagnetic insulator (FMI), antiferromagnetic metal/insulator (AFMM/AFMI), and charge/ orbital order insulator (COI) has been recognized as the intrinsic property of the doped rare earth manganites, represented by general formula $\mathrm{RE}_{1-x} \mathrm{AE}_{x} \mathrm{MnO}_{3}(\mathrm{RE}=\mathrm{La}, \mathrm{Pr}, \mathrm{Nd}$, etc., and $\mathrm{AE}=\mathrm{Ca}, \mathrm{Ba}, \mathrm{Sr}$, etc.). ${ }^{1,2}$ These compounds also exhibit colossal magnetoresistance (CMR), which is now believed to be related intimately to COI state. ${ }^{1,2}$ Therefore the strong phase coexistence and the competition between the COI and FMM phases are now crucial elements in the explanation of CMR. ${ }^{2,3}$ However, the magnetoelectrical properties, particularly the phase coexistence/fluctuation cannot be determined solely by the $3 d$ electron transfer interaction or one-electron bandwidth $(W) .{ }^{1}$ The structural and magnetoelectrical properties can be different even for the identically distorted perovskite lattice structure, the former of which is generally determined by the tolerance factor or the $A$-site averaged ionic radius alone. Attfield and Rodríguez-Martínez ${ }^{3-5}$ conclusively demonstrated that the FM transition temperature $\left(T_{C}\right)$ as well as the metal-insulator transition (MIT) temperature $\left(T_{\mathrm{IM}}\right)$ depends strongly not only on the average ionic radius but also on the size mismatch of the trivalent rare-earth and divalent alkaline-earth ions of the $A$-site of the $\mathrm{ABO}_{3}$ type structure. The size mismatch representing the local lattice distortion transmitted from the randomly substituted $A$-site is measured by the variance, $\sigma^{2}$ $=\Sigma y_{i} r_{i}^{2}-\left\langle r_{A}\right\rangle^{2}$, where $y_{i}$ is the fraction of the $i$ th cation, $r_{i}$ is its radius, and $\left\langle r_{A}\right\rangle$ is the average radius of the cations at the rare earth site. The local distortion arising from the difference in ionic radii, that is, $\sigma^{2}$ and/or the random Coulomb

${ }^{a)}$ Electronic mail: hks65@mail.nplindia.org. potential due to the trivalent/divalent ion mixture is the source of the quenched disorder (QD). Here, QD means the temperature-independent atomic-scale local inhomogeneity producing randomness in potential energy, transfer energy, electron number, exchange interaction, etc. In the hole-doped perovskite manganites, therefore, this kind of QD or the potential randomness is unavoidable except for some special cases. $^{1}$

The size disorder introduces variety of phenomena, such as, metamagnetism and ferromagnetic-metal to cluster glassinsulator transition ${ }^{6,7}$ at $x \approx 0.5$, collapse of the CO-orbital order $(\mathrm{OO})$ state at $x \approx 0.45$ (Ref. 8) and the Griffiths phase $^{9,10}$ in low and intermediate $W$ manganites. The magnetoelectric phase evolution as a function of size disorder $\left(\sigma^{2}\right)$ is expected to strongly influence the low field magnetotransport properties. In the present work we report the effect of size mismatch induced disorder on phase fluctuation and low field magnetoelectrical properties of intermediate $W$ manganite $\mathrm{Nd}_{0.58-x} \mathrm{Gd}_{x} \mathrm{Sr}_{0.42} \mathrm{MnO}_{3}$. The size disorder has been introduced by partial substitution the larger $\mathrm{Nd}^{3+}(r$ $=1.27 \AA)$ by smaller $\mathrm{Gd}^{3+}(r=1.21 \AA)$ cation. The $\left\langle r_{A}\right\rangle$ and $\sigma^{2}$ were calculated using twelve-coordinate ionic radii given by Y. Tokura ${ }^{1}$

\section{EXPERIMENTAL}

Polycrystalline $\mathrm{Nd}_{0.58-x} \mathrm{Gd}_{x} \mathrm{Sr}_{0.42} \mathrm{MnO}_{3} \quad(x \sim 0.0, \quad 0.04$, $0.08,0.12,0.16,0.20,0.25,0.30,0.35$, and 0.42 ) samples were synthesized by the solid state reaction method. The desired amounts of high purity (99.99) $\mathrm{Nd}_{2} \mathrm{O}_{3}, \mathrm{Gd}_{2} \mathrm{O}_{3}$, $\mathrm{SrCO}_{3}$, and $\mathrm{MnCO}_{3}$ were weighed and mixed homogeneously. The thoroughly mixed and ground material was heated at $920{ }^{\circ} \mathrm{C}$ for $24 \mathrm{~h}$ and $1200{ }^{\circ} \mathrm{C}$ for $36 \mathrm{~h}$ with intermediate grinding. Then the powders were pressed in form of rectangular pellets of dimension length $=10 \mathrm{~mm}$, width $=5 \mathrm{~mm}$, and thickness $=0.5 \mathrm{~mm}$ and sintered at $1350{ }^{\circ} \mathrm{C}$ for 
TABLE I. Variation of $T_{C}$ and $T_{\mathrm{IM}}$ with GD content $(x),\left\langle r_{A}\right\rangle$ and variance.

\begin{tabular}{llccc}
\hline \hline $\begin{array}{c}\text { Gd content } \\
(x)\end{array}$ & $\begin{array}{c}\left\langle r_{A}\right\rangle \\
(\AA)\end{array}$ & $\begin{array}{c}\sigma^{2} \\
\left(\times 10^{-3} \AA^{2}\right)\end{array}$ & $\begin{array}{c}T_{C} \\
(\mathrm{~K})\end{array}$ & $\begin{array}{c}T_{\mathrm{IM}} \\
(\mathrm{K})\end{array}$ \\
\hline 0 & 1.3414 & 7.04 & 275 & 223 \\
0.04 & 1.339 & 7.521 & 263 & 213 \\
0.08 & 1.3366 & 7.99 & 248 & 213 \\
0.12 & 1.3342 & 8.448 & 223 & 203 \\
0.16 & 1.3318 & 8.895 & 198 & 188 \\
0.20 & 1.3294 & 9.33 & 168 & 168 \\
0.25 & 1.3264 & 9.857 & 133 & 118 \\
0.30 & 1.3234 & 10.366 & 117 & 105 \\
0.35 & 1.3204 & 10.858 & 108 & 83 \\
0.42 & 1.3162 & 11.516 & 83 & $\cdots$ \\
\hline \hline
\end{tabular}

$24 \mathrm{~h}$. The structural and surface morphological characterization was done by powder x-ray diffraction (XRD) and scanning electron microscopy (SEM). The XRD data was refined by the Reitveld method using FULL-PROF program. The magnetic characterization was carried out by measuring temperature and frequency dependent ac susceptibility of all the samples. The resistivity was measured in four contact configurations in the temperature range of $4.2-350 \mathrm{~K}$ and the MR in magnetic field up to $10 \mathrm{kOe}$ was measured in the range of $77-350 \mathrm{~K}$. The $\mathrm{MR}$ has been defined as $\mathrm{MR}=\left(\rho_{0}\right.$ $\left.-\rho_{H} * 100\right) / \rho_{0}, \rho_{0}$ and $\rho_{H}$ are resistivity measured at zero and magnetic field $H$, respectively.

\section{RESULTS AND DISCUSSION}

Since $\mathrm{Gd}$ is smaller than $\mathrm{Nd}$, the average $A$-site cationic radius $\left\langle r_{A}\right\rangle$ decreases, while $\sigma^{2}$ increases. The relationship between Gd concentration $x,\left\langle r_{A}\right\rangle$, and $\sigma^{2}$ are linear. Structural characterization by XRD reveals that all the samples are single phase and have very good crystallinity. Refinement of the XRD data carried out by the Reitveld method reveals that all samples have orthorhombic structure with space group Pbnm and the lattice parameters are $a>b>c\left(\mathrm{O}^{\prime}\right.$ type orthorhombic). Within the limit of the instrumental error the " $a$ " parameter is observed to decrease slightly with increasing $x$, while " $b$ " and " $c$ " remain nearly constant. From the variation trend of the lattice parameters a gradual decrease in orthorhombicity is observed with increasing $x$. This could be understood in terms of intrinsic pressure in the orthorhombic lattice due to the substitution of smaller $\mathrm{Gd}^{3+}(r=1.21 \AA)$ cations for the larger $\mathrm{Nd}^{3+}$. However, the variation in only one lattice parameter reflects the unidirectional nature of the pressure, which could be related to some specific/selective ordering of the $\mathrm{Gd}^{3+}$ cations in the parent $\mathrm{Nd}_{0.58} \mathrm{Sr}_{0.42} \mathrm{MnO}_{3}$ unit cell. SEM results show that the surface of these samples generally consists of spherical grains and well defined grain boundaries. The average grain size is found to be $\sim 1 \mu \mathrm{m}$.

The paramagnetic-ferromagnetic (PM-FM) transition temperature $\left(T_{C}\right)$ is observed to decrease with increasing $x / \sigma^{2}$ (see Table I). In the pristine sample $(x=0), \sigma^{2}$ $=0.00704 \AA^{2}$, and $T_{C}=275 \mathrm{~K}$, which was found to gradually decrease as a function of increasing $\sigma^{2}$ to $\approx 82 \mathrm{~K}$ for $x$ $=0.42$. The representative $\chi-T$ curves corresponding to $x$ $=0.00,0.04,0.12,0.25$, and 0.35 along with the $\sigma^{2}$ values

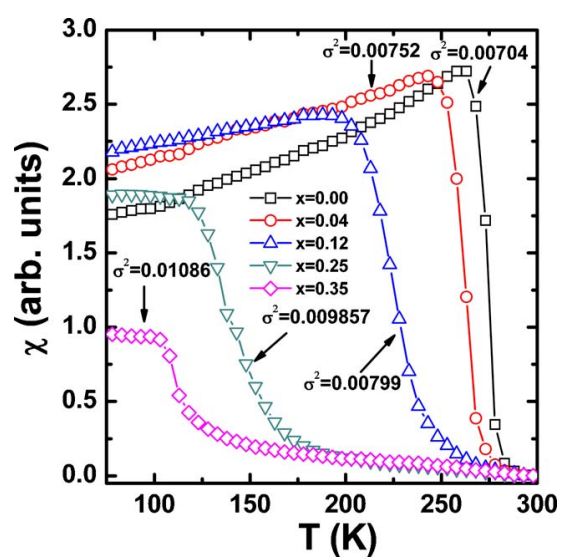

FIG. 1. (Color online) Variation in ac susceptibility $(\chi)$ as a function of temperature for $\mathrm{Nd}_{0.58-x} \mathrm{Gd}_{x} \mathrm{Sr}_{0.42} \mathrm{MnO}_{3}$.

are plotted in Fig. 1. In addition to this appreciable broadening of the PM-FM transition was observed at higher $\sigma^{2}$ and $x$. Thus Gd substitution at the $\mathrm{Nd}$ site has the twin effects on the PM-FM phase transition. The $T_{C}$ suppression and concomitant increase in the transition width with increasing $x / \sigma^{2}$ is explained in terms of the structural and microstructural changes. Smaller $\mathrm{Gd}^{3+}$ cations causes a decrease in $\left\langle r_{A}\right\rangle$ and in addition it also increases $\sigma^{2}$. The decrease in $\left\langle r_{A}\right\rangle$ coupled with the enhanced size disorder $\left(\sigma^{2}\right)$ causes a local deformation of the $\mathrm{MnO}_{6}$ octahedra, which strengthens the Jahn-Teller (JT) distortion that increases carrier localization due to reduced bandwidth. ${ }^{3-5}$ Simultaneously, the smaller values of $\left\langle r_{A}\right\rangle$ results in a decrease in the $\mathrm{Mn}-\mathrm{O}-\mathrm{Mn}$ bond angles, which weakens the FM-DE (double exchange) of the $e_{g}$ electrons. The usual broadening of the FM transition with increasing $x$ is due to the successive lowering of the $\mathrm{Mn}-$ $\mathrm{O}-\mathrm{Mn}$ bond angles. Thus decrease in $\left\langle r_{A}\right\rangle$ (increase in $\sigma^{2}$ ) is expected to enhance the competition between the two coexisting phenomena, viz., the JT distortion and the FM-DE (Refs. 3-5) and hence may cause strong phase fluctuation at or in the vicinity of a critical value of $\left\langle r_{A}\right\rangle / \sigma^{2}$. ${ }^{1}$ Since carrier localization is explicitly reflected in electrical transport measurement, the nature of the temperature dependent resistivity is expected to change in the vicinity of the critical $\left\langle r_{A}\right\rangle / \sigma^{2}$.

The IMT of the Gd free sample is $T_{\mathrm{IM}} \approx 223 \mathrm{~K}$ and like the $T_{C}$ it is also gradually observed to decrease with increase in $x / \sigma^{2}$. The $T_{\mathrm{IM}}$ of $x=0.35$ sample was $\sim 83 \mathrm{~K}$ and the $x$ $=0.42$ sample did not show IMT at all. The variation in IMT as a function of $\sigma^{2}$ is listed in Table I. However, unlike the PM-FM transition which broadened with increasing $x / \sigma^{2}$, the IMT was to observe sharpen. The sharpening of the IMT is clearly visible in the $\rho$-T curves presented in Fig. 2 .

In the pristine sample $\left(\sigma^{2}=0.00704 \AA^{2}\right)$, the difference between $T_{C}$ and $T_{\mathrm{IM}}$ is large $\sim 50 \mathrm{~K}\left(T_{C}>T_{\mathrm{IM}}\right)$. At $\sigma^{2}$ $=0.00933 \AA^{2}(x=0.20)$, both $T_{C}$ and $T_{\mathrm{IM}}$ attain the same value and at further higher variances the difference between them again increases slightly $\left(T_{\mathrm{IM}}<T_{C}\right)$. In polycrystalline manganites, the difference between the $T_{C}$ and $T_{\mathrm{IM}}$ is generally attributed to the grain boundary $(\mathrm{GB})$ disorder. ${ }^{11-13}$ Therefore, at lower values of $\sigma^{2}$ the difference between $T_{C}$ and $T_{\mathrm{IM}}$ is due to the GB disorder. However, near equality of $T_{C}$ and $T_{\mathrm{IM}}$ at intermediate values of $\sigma^{2}$ could be understood 


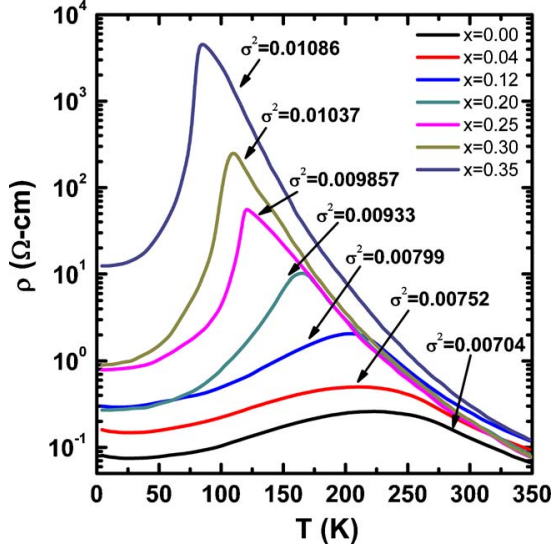

FIG. 2. (Color online) Variation in resistivity as a function of temperature of $\mathrm{Nd}_{0.58-x} \mathrm{Gd}_{x} \mathrm{Sr}_{0.42} \mathrm{MnO}_{3}$. The corresponding variances $\sigma^{2}$ (in $\AA^{2}$ ) have been indicated.

in terms of the QD due to the size mismatch. As the size disorder increases the QD induces phase fluctuation and enhanced phase competition. The competing phases in the presented case are expected to be FM-metal and AFM-CO-insulator. ${ }^{1}$ Consequently, the competition between these two disorders, viz., the GB disorder and the QD is responsible for the observed variation in $T_{C}$ and $T_{\mathrm{IM}}$. At lower values of $x$, e.g., the $\mathrm{Gd}$ free sample the large difference in $T_{C}$ and $T_{\mathrm{IM}}$ shows that the GB disorder dominates over the QD measured by $\sigma^{2}$. As $x$ increases the GB disorder remains unaffected while the value of $\sigma^{2}$ increases and enhances the phase fluctuation. Since $\mathrm{Nd}_{0.58} \mathrm{Sr}_{0.42} \mathrm{MnO}_{3}$ has a FM-M ground state while $\mathrm{Gd}_{0.58} \mathrm{Sr}_{0.42} \mathrm{MnO}_{3}$ is an AFM-CO insulator, the partial substitution of $\mathrm{Nd}$ by $\mathrm{Gd}$ could result in creation of AFM-I/CO-I phase in the parent FM-M matrix and hence enhance phase fluctuations, which are expected to increase with increasing Gd content and maximize around intermediate values of $x$, e.g., $x=0.20-0.30$. So at these $\mathrm{Gd}$ concentrations the phase fluctuations is expected to be the maximum and dominate over the GB disorder resulting in near equality of $T_{C}$ and $T_{\mathrm{IM}}$. Near the highest value of $\sigma^{2}$ the $T_{\mathrm{IM}}$ becomes smaller than the corresponding $T_{C}$. This could be due to the appearance of some AFM-CO-OO cluster in the FM matrix.

The variation in MR $(H=3 \mathrm{kOe})$ as a function of temperature is shown in Fig. 3. It is well known that the MR in polycrystalline samples has two contributions: (i) the intrinsic component that arises due to the FM-DE and peaks around $T_{C} / T_{\mathrm{IM}}$ and (ii) the extrinsic part that is determined by the grain boundaries and increases monotonously as the temperature is lowered. ${ }^{11-13}$ As seen in the Fig. 3, with increasing $x$ and hence $\sigma^{2}$, the MR is observed to increase. The intrinsic MR is observed to increase from $\sim 3 \%$ to $35 \%$ as the variance increases from 0.00704 to $0.009857 \AA^{2}$ and then decreases for larger values of $\sigma^{2}$. In contrast, the extrinsic GB part remains nearly constant and then increase slightly, thus it has a weak dependence on the $\sigma^{2}$. The variation in the peak MR measured at 3 and $10 \mathrm{kOe}$ and MR at $T=77 \mathrm{~K}$ is shown in the inset of Fig. 3. The intrinsic MR that shows a maximum around $T_{C} / T_{\mathrm{IM}}$ is expected to be the highest in the vicinity of the maximum phase fluctuation. To

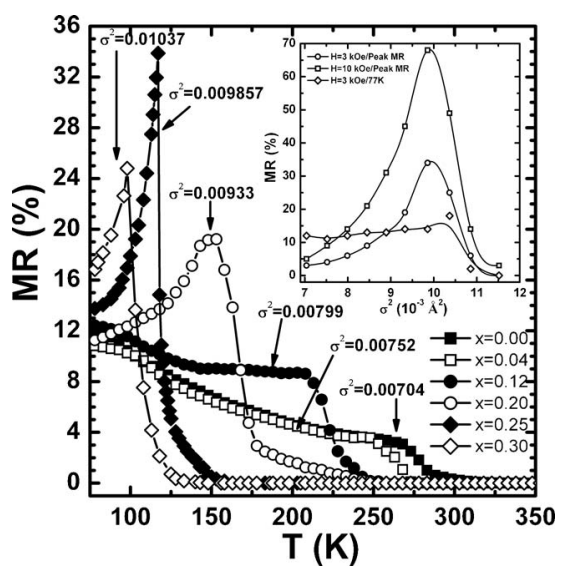

FIG. 3. Temperature dependence of MR measured at magnetic field $H$ $=3 \mathrm{kOe}$. The inset shows the variation in peak MR $(\%)$ with $\sigma^{2}$ (with unit $\AA^{2}$.

verify this we measured the temperature dependent MR at different magnetic fields for all the samples. The variation in peak MR (maximum MR around $T_{C} / T_{\mathrm{IM}}$ ) with $\sigma^{2}$ shows that maximum low field $\mathrm{MR} \approx 35 \%$ at $H=3 \mathrm{kOe}(68 \%$ at $H$ $=10 \mathrm{kOe})$ is centered around $\sigma^{2}=0.009857 \AA^{2}(x=0.25)$.

\section{CONCLUSIONS}

We have studied the effect of the quenched disorder arising from the size disorder (measured by the variance $\sigma^{2}$ ) at the RE site in polycrystalline $\mathrm{Nd}_{0.58-x} \mathrm{Gd}_{x} \mathrm{Sr}_{0.42} \mathrm{MnO}_{3}$. Although both $T_{C}$ and $T_{\mathrm{IM}}$ are observed to decrease with increasing $\sigma^{2}$ the former becomes broad while the later is sharpened. This feature is attributed to phase fluctuation due to the size disorder caused by Gd doping. The large difference in the $T_{C}$ and $T_{\mathrm{IM}}\left(T_{C}>T_{\mathrm{IM}}\right)$ at lower values of $\sigma^{2}$ suggests the dominance of the GB disorder over the quenched disorder/phase fluctuation. Gradual sharpening of the IMT with increasing $\sigma^{2}$ is followed by very sharp metallic transition around $\sigma^{2}=0.009857 \AA^{2}$. At further higher $\sigma^{2}$ the $T_{\mathrm{IM}}$ again becomes smaller than $T_{C}$ and IMT finally vanishes. Large low field MR observed in the vicinity of $\sigma^{2}$ $=0.009857 \AA^{2}$ demonstrates the importance of quenched disorder/phase fluctuation in manganites.

\section{ACKNOWLEDGMENTS}

M.K.S. and R.P. thankfully acknowledge CSIR and UGC-New Delhi for award of research fellowship.

${ }^{1}$ Y. Tokura, Rep. Prog. Phys. 69, 797 (2006).

${ }^{2}$ E. Dagotto, New J. Phys. 7, 67 (2005).

${ }^{3}$ L. M. Rodríguez-Martínez and J. P. Attfield, Phys. Rev. B 54, R15622 (1996)

${ }^{4}$ J. P. Attfield, Chem. Mater. 10, 3239 (1998).

${ }^{5}$ L. M. Rodríguez-Martínez and J. P. Attfield, Phys. Rev. B 63, 024424 (2000).

${ }^{6}$ R. D. Shannon, Acta Crystallogr. 32, 751 (1976).

${ }^{7}$ K. R. Mavani and P. L. Paulose, Appl. Phys. Lett. 86, 162504 (2005).

${ }^{8}$ K. F. Wang et al., Appl. Phys. Lett. 88, 152505 (2006).

${ }^{9}$ K. F. Wang et al., Appl. Phys. Lett. 89, 222505 (2006).

${ }^{10}$ N. Rama et al., Phys. Rev. B 70, 224424 (2004).

${ }^{11}$ A. Gupta et al., Phys. Rev. B 54, R15629 (1996).

${ }^{12}$ X. L. Wang et al., Appl. Phys. Lett. 73, 396 (1998).

${ }^{13}$ P. K. Siwach et al., J. Phys.: Condens. Matter 20, 273201 (2008). 